\title{
Does Creating an Artificial General Intelligence Require General Collective Intelligence in Order to be Reliably Achievable?
}

Andy E. Williams, Nobeah Foundation, Nairobi, Kenya

\begin{abstract}
General Collective Intelligence or GCI has been predicted to create the potential for an exponential increase in the problem-solving capacity of the group, as compared to the problem-solving capacity of any individual in the group. A functional model of cognition proposed to represent the complete set of human cognitive functions, and therefore to have the capacity for human-like general problem-solving ability has recently been developed. This functional model suggests a methodical path by which implementing a working Artificial General Intelligence (AGI) or a working General Collective Intelligence might reliably be achievable. This paper explores the claim that there are no other reliable paths to AGI currently known, and explores why this one known path might require an exponential increase in the general problem-solving ability of any group of individuals to be reliably implementable. And why therefore, AGI might require GCI to be reliably achievable.
\end{abstract}

\section{Introduction}

As described by the recently defined Functional Modeling Framework (FMF) [1], which in turn leverages the recently defined approach of Human-Centric Functional Modeling or HCFM [2], humanlike general problem solving ability can potentially be represented by a functional model in which the cognitive system navigates a space of concepts (a conceptual space). In this model, the cognitive system must have some cognitive awareness process to detect the concepts currently in the awareness of the cognitive system, and to choose which cognitive processes (reasoning or understanding processes) to execute in order to navigate the conceptual space from any general problem, to the solution to that problem.

Representing the cognitive processes (reasoning or understanding processes) of an Artificial General Intelligence as being AI solutions that solve specific problems [3], then based on the same functional model, achieving human-like general problem-solving ability in an Artificial General Intelligence requires the AGI to have the ability to use some combination of available AI solutions, or to create new AI solutions, to solve any given problem within human capacity to solve, where that problem is defined in any given context within human capacity to understand.

From the functional modeling perspective problems are defined as the lack of a path from a starting point in the functional state space of the cognitive system (the conceptual space) to an ending point. Solutions are defined in terms of a set of basic functions that spans the entire conceptual space, as well as the interactions between these functions. A direct interaction between functions is a first order interaction. An interaction between two interactions is a second order interaction. There is a limit to the order of interaction that an individual human cognition has the capacity to conceptualize. Problems or solutions of higher order complexity are problems that cannot be reliably defined, or solutions that cannot reliably be discovered, within the capacity of individual human cognition. The question is, as the number $\mathrm{N}$ of AI solutions increases, whether finding the best combination of available AI solutions to solve any given problem in any given context at some point become a problem of higher order complexity that is not reliably solvable with any solution within the capacity of individual human cognition to design, or with any solution within the current capacity of groups of humans to design? If so then the problem of gaining the ability to use the best combination of available AI solutions might not be reliably solvable with any currently available solution. If General Collective Intelligence has the capacity to significantly increase the general problem-solving ability of a group, and therefore to 
significantly increase the complexity a group can navigate, General Collective Intelligence might in that case be required to give groups the capacity to solve this problem, assuming the order of complexity is not so high that it is also outside the capacity of a GCI.

To explore this question, first define a functional model of the AGI as consisting of a set of functions from which any function or process of the AGI might be composed. Any problem of understanding the AGI can then be defined as the problem of finding a path from one functional state of the AGI to another functional state. And any solution can be defined as such a path expressed in terms of those functions, or in terms of interactions between those functions. Again, an interaction of order $\mathrm{N}$ between functions is considered "higher order" if $\mathrm{N}$ is greater than human beings individually or collectively (without GCI) have the capacity to reliably conceive, so that human beings are not reliably capable of perceiving the problem, or reliably capable of detecting the solution at a sufficiently specific level for any individual or group to confirm the definition of that problem as valid, or for any individual or group to be able to implement that solution and therefore for any individual to be able to confirm the validity of the solution through experimentation.

From the perspective of the cognitive model referred to in this paper [1], the statement: "fit inside the cognition of an individual human" is objectively defined as a problem or solution that fits within the maximum volume of "conceptual space" an individual human cognitive system might have access to.

\section{Functional Modeling Makes All Conceptual Space Navigable but not Necessarily Accessible}

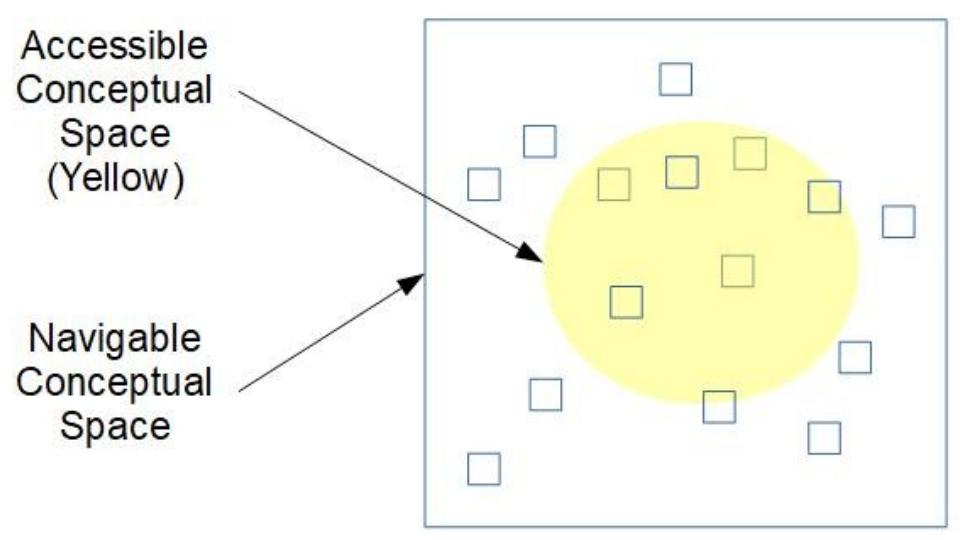

Figure 1: The conceptual space that can potentially be navigated might be unbounded (blue square). The conceptual space that is actually accessible by any individual cognition is suggested to be bounded (yellow square).

Problems that are too big for any individual human cognition start or end at points in conceptual space which don't fit within this volume, and therefore can't be defined by any individual human cognition. 


\section{Undefinable Problems}
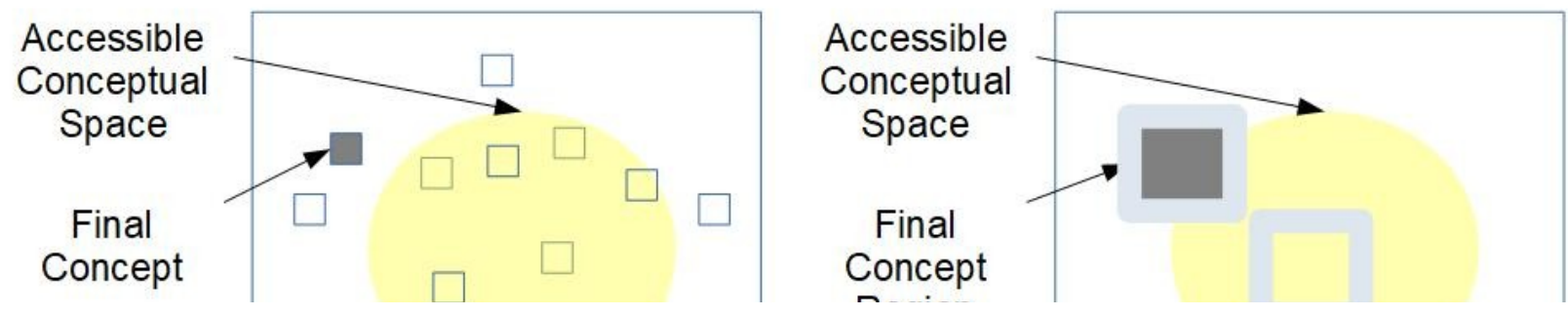

Similarly, solutions that are too big for individual human cognition cannot be discovered.

\section{Undiscoverable Solutions}
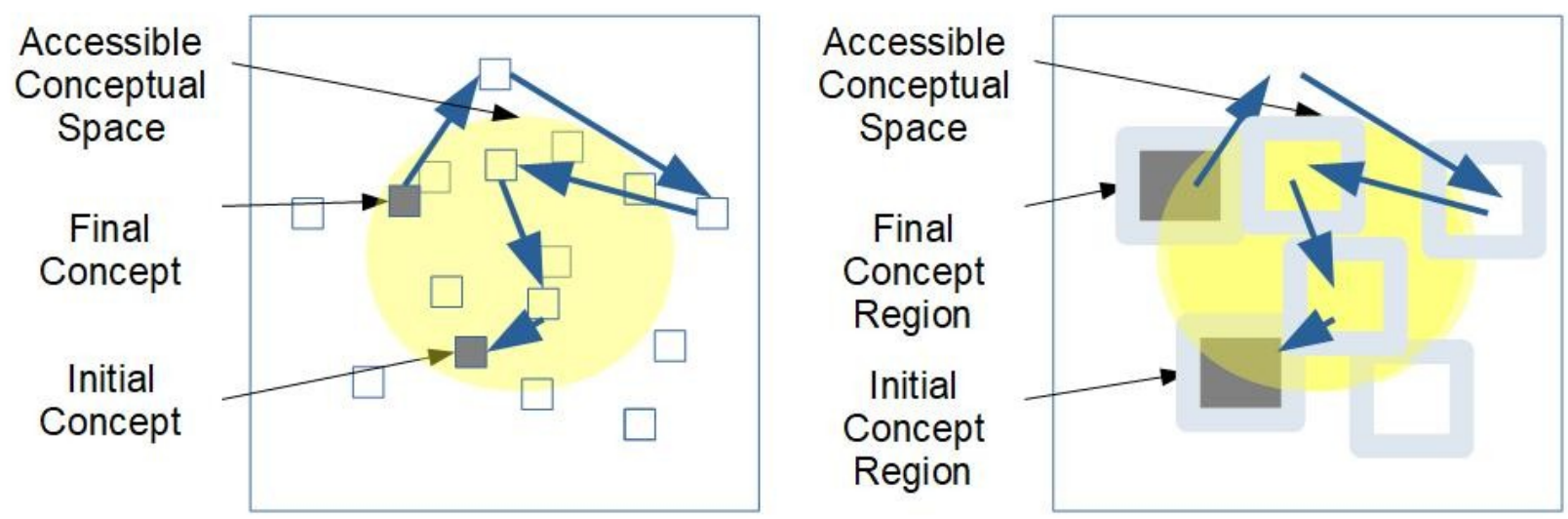

Figure 3: Solutions are undiscoverable if the required reasoning exits accessible conceptual space (left), or if the initial, final, or intermediate concepts in reasoning are not sufficiently revolvable to be located within the accessible region (right).

Of course humans can always increase the degree to which they generalize a problem, or increase the degree to which they generalize solutions to that problem, so that problems of any level of complexity can reliably be defined at some level that is general enough to fit inside the cognition of an individual human, so that solutions of any level of complexity can reliably be discovered at some level general enough to fit inside such a cognition [4].

However, increasing the level of generalization at which problems are conceptualized so that it becomes possible to communicate higher order problems or higher order solutions, by definition, implies that defining the problem at a specific enough level for any individual or group to confirm the definition of that problem as valid, or defining the solution as a specific enough level for any individual or group to confirm the solution through implementation, is not reliably achievable. In simple terms, 
new concepts require existing concepts to anchor to. When new concepts are sufficiently complex, no common set of anchor points can reliably be assumed to be present. A complex concept like an implementation of the functional model of AGI requires a great many anchors that can't generally assumed to be present. Communicating an understanding is equivalent to helping someone navigate from one set of conceptual anchor points to another in terms of objectively provable steps. Because no one can know what is in another's head, concepts like this AGI are complex enough that they can only be communicated in terms of generalizations broad enough to anchor to simple conceptual reference points that can be assumed to be present. Because these generalizations must be broad enough to eliminate the potential for misunderstanding in most, they are also broad enough to eliminate fine distinctions, so that most will not understand how those generalizations apply to any specific aspect of the problem of implementing an AGI. Reasoning expressed in terms of broad generalizations might be justified by a great many concrete observations, but when that number of justifying observations is sufficiently large, the totality of those observations, and therefore justification of the generalization they support, cannot reliably be communicated. As a result, in the case of sufficiently complex concepts, which higher order systems like an implementation of AGI are by definition, no one individual can reliably communicate that concept in a way that people can reliably understand. Therefore no one individual can reliably communicate that concept in a way that people reliably understand in sufficient numbers to reliably convince all the roles required to implement an AGI.

From the perspective of the cognitive model referred to in this paper [1], the level of simplification involved in any generalization is equivalent to a reduction in the order of complexity of the concept.

\section{Reduction in Order of Complexity of a Problem and of the Concept Representing it}
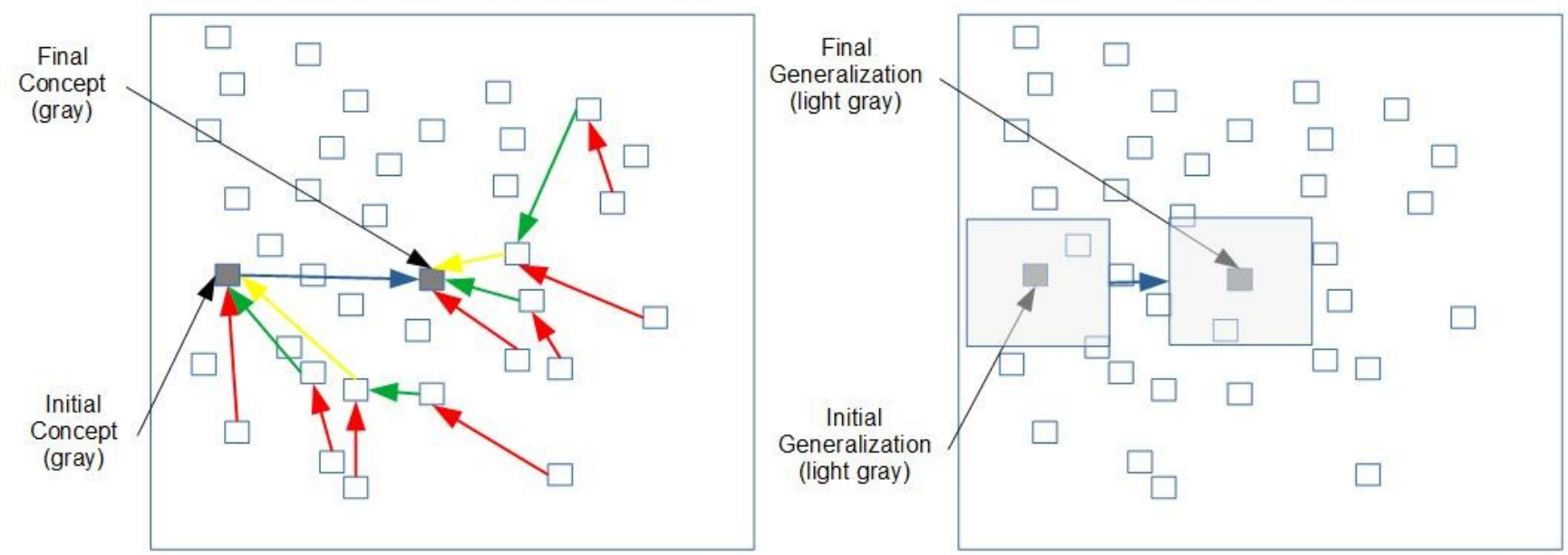

Figure 4: On the left, first order reasoning processes (red) define problems with first order solutions (green). The interaction between multiple first order reasoning processes (green) defines problems with second order solutions (yellow). The interaction between multiple second order reasoning processes (yellow) defines problems with third order solutions (blue). On the right, higher order problems might not be definable or solvable at a specific level (at the level of the cognitive system's resolution in conceptual space), but generalizations of such higher order problems or solutions can always potentially be found. 
In communicating a concept there is a speaker and a listener. The speaker might have come to believe a certain generalization is true because it being true is consistent with all of their observations. But as the complexity of the concept increases, and therefore the level of simplification involved in the generalization increases, the number of observations required for the listener to accept the generalization as being true rises, and a point is reached at which the truth of the generalization cannot reliably be communicated to that listener.

If implementing an AGI is a higher order problem, a generalization that simplifies a model of AGI to the point that it is reliably understandable, might then be a generalization whose truth cannot then be reliably communicated without a system such as GCI that significantly increases the ability of a group to communicate truth.

But assuming the model of AGI being discussed is valid, and assuming that somehow it has been communicated to sufficient people that all the roles required to implement it have been filled, the question then becomes under what conditions is implementing the model reliably achievable? In order for an outcome to be reliably achievable by any given process, that process must be able to scale the magnitude of that outcome until the targeted magnitude of outcome has a greater than random probability of being achieved. One way to scale outcomes is to take a process that produces some magnitude of the desired outcome, and repeat that process over time. Another way to scale outcomes is to take a process that produces some magnitude of the desired outcome within a group of a given size, and to scale the size of the group. Consider the problem of designing an AGI capable of navigating a library AI solutions in order to increase it's general problem solving ability. This AGI must have some model for identifying the function of each AI solution.

Assume that it is possible for an individual or group to create a simple functional model of the overall outcome that each AI solution functions to achieve. Then if there are $\mathrm{N}$ models, and $\mathrm{M}$ modeling approaches, the total number of models that must be created to ensure a model exists in whatever modeling methodology the AGI designer has chosen is $\mathrm{M}^{*} \mathrm{~N}$. If the " $\mathrm{i}$ "th model consists of $\mathrm{L}_{\mathrm{i}}$ components that can also be modeled, then the total number of models to be navigated is $\mathrm{L}_{\mathrm{i}}^{*} \mathrm{M} * \mathrm{~N}$. If the number of AI approaches (deep learning, reinforcement learning, NLP, decision tree, etc.) is K, and the number of implementations of each approach for the "i" $\mathrm{i}$ "th function is $\mathrm{N}_{\mathrm{Ki}}$, then the total number of models is now the sum of $\mathrm{N}_{\mathrm{ki}}{ }^{*} \mathrm{~L}_{\mathrm{i}}^{*} \mathrm{M} * \mathrm{~N}$ for all "i".

As each new AI solution " $\mathrm{j}$ " is created, having the ability to navigate all available AI solutions implies that whatever solution the AGI begins with, it can always find the best solution for a given purpose among all the AI solutions available. "Best" implies a comparison, and for such a comparison to be universally able to be made, the relationship of that new solution to all other solutions " $i$ " must be defined in every context $\mathrm{C}$ where any of those solutions might be used. This comparison information must itself be modeled if it is to reliably be navigated. Each new AI solution then potentially increases modeling tasks by the sum of $\mathrm{N}_{\mathrm{ki}}{ }^{*} \mathrm{~L}_{\mathrm{i}}{ }^{*} \mathrm{M} * \mathrm{~N}$ for all "i".

Human-centric functional modeling is modeling in terms of intrinsic human meaning, rather than having meaning that requires some interpretation that is separate from the model itself. Modeling concepts in the "conceptual space" defined by the FMF, in combination with this cognitive model that gives "intrinsic human meaning" to constructs modeled in this space, is proposed to be equivalent to semantic modeling, which by consensus in the expert community has not been fully implemented by any other existing approach to semantic modeling [18]. 
However, neither this conceptual space nor an AGI representing this cognitive model have yet been implemented, therefore the capacity for complete semantic modeling cannot be assumed to exist. Since complete semantic modeling capability has not yet been implemented, then all current semantic models rely on some degree of human interpretation, and therefore store part of their meaning in that interpretation rather than completely in the model. So by definition, if human interpretation cannot be automated, and we assume that without AGI this is the case, then semantic modeling tasks cannot yet be automated either.

If automating semantic modeling requires a solution of higher order complexity like an AGI, then by definition it is a higher order problem. If it is a higher order problem, then any generalization that simplifies the problem to the degree that it can be reliably be solved, such as a generalization that reduces the number of modeling tasks to the point that they are reliably achievable, is a generalization that might not reliably be communicated without a higher order solution like GCI.

If these semantic modeling tasks cannot yet be automated, and they cannot be generalized in some way that reduces the number of tasks significantly, at some point the number of modeling tasks required to implement the current functional model of AGI might rise to the point that they cannot reliably be performed by any individual or current group without GCI. The question is whether designing an AGI with human-like general problem-solving ability require an ability to perform more modeling tasks than this cutoff point.

To explore this question, firstly, humans solve cognitive problems through reasoning. Human-like reasoning ability must entail the capacity to use any reasoning process used by any human at any time. If the claim that this model of cognition has human-like general problem-solving ability is too complex to prove in general, because it is a higher order problem, then having the capacity to solve each specific problem must be proven for every specific problem mankind has every conceptualized. The reasoning used by any human at any time includes at minimum all the reasoning ever defined in any media that ever existed. Where writing found on the pages of websites might be intuitively recognizable as being media, sign language that is in essence written in the air, might not be. Both must be included. From a Human-Centric functional Modeling (HCFM) point of view, the most important definition of media is anything that functions to transmit meaning. This includes any meaning ever recorded in memory and transmitted through words, movement, or other forms that might be considered as the media of "this existence".

A generalization might be simple enough to be provable, but such a generalization must first be applied through some process. No universal semantic model currently exists, so no universal semantic model of a process of generalization can exist. If no universal semantic model for a process of generalization exists, then no method for automating the creation of those generalizations can exist. If each reasoning process cannot be generalized through some automated process, then validating that an AGI is capable of each reasoning process requires that each specific reasoning process be represented in an AI solution, so that with that AI solution the AGI can be confirmed to have the capacity for that specific reasoning process. One way to do so might be to confirm some AI solution can produce the same conclusion as the human reasoning within the same context. And to test this for all recorded reasoning in all recorded contexts. This is a large task.

The number of people on the earth is 7.594 billion (2018). Any design or other task that is dependent on the size of the group, and that without GCI cannot reliably be achieved by 7.594 billion people, is a task that cannot reliably be performed by any individual or group without GCI. The distribution of intelligence among people on the earth is a normal distribution. Any design or other task that without 
GCI is dependent on a single individual or group of individuals having cognitive ability that is above the level that statistically can reliably occur in a group of 7.594 billion people with a normal distribution of cognitive ability, is a task that cannot reliably be performed by any individual or group without GCI. Any design or other task that without GCI requires a greater amount of resources to incentivize the required number of participants, than any individual or group can reliably be incentivized to provide, is a task that can not reliably be performed by any individual or group without GCI. Any design or other task that without GCI cannot be decoupled into small enough components that the number of participants can be scaled to the point that it is reliably achievable, is a task that cannot reliably be performed by any individual or group without GCI.

Assume that a semantic model of each AI solution takes 10 minutes to define, then the entire population of the earth together is potentially capable of creating 75.94 billion models for reasoning processes every ten minutes. However, the required capacity to manually model reasoning as a function of the number of people, at best rises linearly with the number of people. As the number of reasoning processes being created every ten minutes rises, without GCI to provide simplifying generalizations that can reliably be communicated, there must always be some point at which the required capacity to manually model reasoning cannot be achieved without the capacity to introduce some simplification that exponentially decreases the modeling that must be performed. If modeling reasoning above this point cannot reliably be achieved within human cognitive abilities, then this modeling is a higher order problem requiring a higher order solution.

Specifically, if the cognitive system requires the capacity to compare any two reasoning processes in order to have the capacity to reliably navigate all reasoning processes, and if each reasoning process must be manually modeled in order to be compared, then the required capacity to model the relationships between reasoning rises as some exponent of the number of reasoning processes. The precise number is proposed to be the square of the sum of $\mathrm{N}_{\mathrm{ki}}{ }^{*} \mathrm{~L}_{\mathrm{i}}{ }^{*} \mathrm{M} * \mathrm{~N}$ for all " $\mathrm{i}$ ".

Assuming that $\mathrm{N}_{\mathrm{ki}}{ }^{*} \mathrm{~L}_{\mathrm{i}}{ }^{*} \mathrm{M}=1$, the required number of reasoning relationships that must be created for every new concept added to the conceptual space reduces to $\mathrm{N}^{2}$. The capacity to create 75.94 billion relationships between functional models every ten minutes in order to give an AGI the capacity to navigate a conceptual space with one new reasoning process added every ten minutes, is sufficient to allow the conceptual space to be navigable with general problem-solving ability only if the number of models of reasoning processes is the square root of 75.94 billion $(870,000)$ or lower. In other words, any solution (such as an AGI in this case) requiring a group to create more than 870,000 relationships every ten minutes between functional models of reasoning processes implemented by AI solutions, is a solution that cannot reliably be achieved. Of course, the assumption that every individual on earth could be dedicated to this modeling full-time, and would also have the aptitude for it, as well as the assumption that $\mathrm{N}_{\mathrm{ki}}{ }^{*} \mathrm{~L}_{\mathrm{i}} * \mathrm{M}=1$, results in the current actual capacity for such functional modeling and for defining relationships between such functional models potentially being much lower.

Higher order problem-solving systems like GCI can reliably solve higher order problems given three assumptions. The first is that an Nth order problem can be decoupled into $\mathrm{N}$ first order problems that are solvable by individuals as lower order problem-solving systems. The second is that the higher order system can introduce a layer of abstraction that enables those $\mathrm{N}$ lower order problems to be generalized so that their solutions might be shared by $\mathrm{N}$ problems already being solved. The third is that the number of problem-solving components in the system (in the case of GCI this is the number of individuals) are greater than $\mathrm{N}$. 
One potential solution to this problem of increasing capacity to manually model reasoning so that designing an AGI is reliably achievable is the combination of General Collective Intelligence (GCI) and Human-Centric Functional Modeling (HCFM). HCFM provides a universal human-centric approach for defining functional models, and defines a minimal set of constructs with which all functional models might be composed, in order to exponentially reduce the number of functional models that must be defined. The Functional Modeling Framework (FMF) applies this approach to modeling the cognitive system. GCI defines an adaptive problem-solving process by which groups can organize themselves in order to gain the capacity to vastly increase their problem-solving ability, and therefore to gain the capacity to vastly increase the complexity of problems they can reliably define, and the complexity of solutions they can reliably discover. Applying GCI to the process of implementing a functional model of AGI is intended to enable all approaches towards AI, and other all approaches to AGI to be decoupled into functional components that might be combined into a single meta-model of AGI that reliably converges on the functionality required for a working AGI, and that does so at vastly increased speed and scale.

\section{Size of the Conceptual Space Required for Human-Like General Problem-Solving Ability}

The actual number of reasoning processes that must be modeled in order to have human-like general problem-solving ability might determine whether this number is within the capacity of groups without GCI. This number might potentially be estimated from the possible combinations of words in a given human language, and from the assumption that any string of words beyond a certain length will repeat reasoning that has already been defined. As an example, the combination of words "The ball on the left is blue and the ball on the right is red", has a different order than the combination of words "The ball on the right is red and the ball on the left is blue." But semantically both strings are the same.

According to some sources [6], the average active vocabulary of an adult English speaker is around 20,000 words, while his passive vocabulary is around 40,000 words. An active vocabulary consists of the words we have learned and actually use when speaking or writing. On the other hand, a passive vocabulary refers to words we've assimilated but have not been able to use.

As an upper boundary, of the reasoning processes that need to be modeled, assume that the length of any reasoning is $\mathrm{n}$ concepts, each represented by a word, and assume that any combination of $\mathrm{n}$ words has some meaning, then the number of possible reasoning processes in a conceptual space containing $\mathrm{n}$ concepts ( $\mathrm{n}$ words) is potentially given by $\mathrm{n}$ !, which can be estimated using Stirling's approximation [7]:

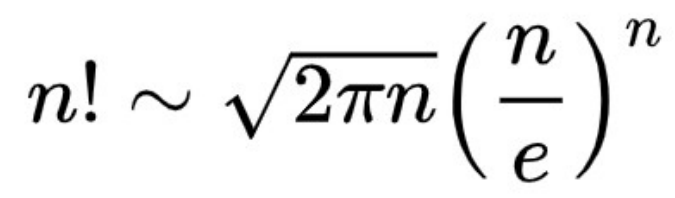

If $\mathrm{n}$ is of the order of $10^{4}$, then $\mathrm{n}$ ! is of the order of $10^{10,0002}$. This is a very large number. If reasoning processes must be modeled manually, this would potentially require all the approximately $10^{10}$ human beings on earth to model $10^{9,9992}$ reasoning processes each. If modeling each reasoning process takes 100 seconds, then all the people on earth modeling reasoning processes for all of the average lifespan of $10^{7}$ seconds could only model $10^{5}$ reasoning processes each. 
Of course, $\mathrm{AI}$ is required only for intuitive reasoning processes. Rational methodical reasoning might be used to connect some exponentially large number of concepts with a very small number of basic reasoning operations. The ability to generalize combined with use of rational methodical reasoning to combine reasoning processes could reduce the number of reasoning processes required to navigate this conceptual space exponentially. For example, four arithmetic operations (addition, subtraction, multiplication, division) on a number system with a base of ten numbers (0 to 9$)$, results in the ability to navigate from the concept of any real number in the infinite set of real numbers to every other real number. Considering the navigation from the concept of each number to the concept of each other number to a reasoning process, because this number of reasoning processes is infinite, it is a far larger set of reasoning processes than the upper bound to the number of reasoning processes required to navigate between all words in the typical English vocabulary. Yet it can be spanned by only four reasoning operations.

The Functional Modeling Framework (FMF) reduces all reasoning or understanding to being a composition of four basic processes. And the approach of Human-Centric Functional Modeling (HCFM) reduces any reasoning or understanding about any entity to be a composition of a minimal set of concepts representing the functions of that entity. The combination of FMF and HCFM might likewise exponentially reduce the set of reasoning required to describe any entity. The takeaway is that anyone in a group might provide a system for navigating the conceptual space that exponentially increases capacity to navigate that conceptual space. In order to maximize the likelihood that such a system is adopted by the group, there must be a mechanism for determining the value of such a system. And there must be a mechanism for expanding the size of the group, and for expanding the problem and solution spaces the group searches, until it reliably contains such a system.

\section{Validating the Concept of a Minimal Conceptual Space for Human-Like Cognition through Observation of Child Development}

If human languages convey concepts and relationships between them with a degree of generalization sufficient to allow the exponential growth in the conceptual space that is predicted to occur as a phase change from animal to human intelligence [5], then a cognitive system with sufficient capacity for generalization as required for this exponential growth might be expected to show evidence of this exponential growth in its conceptual space at some developmental stage. Taking the vocabulary of a child as a rough measure of the size of its conceptual space, that exponential growth appears to be observed:

\begin{tabular}{|l|l|}
\hline Age (In Years) & Vocabulary (Words) \\
\hline 1 to 1.5 & 20 \\
\hline 2 & $200-300$ \\
\hline 3 & $900-1000$ \\
\hline 4 & $1500-1600$ \\
\hline 5 & $2100-2200$ \\
\hline 6 & 2600 (expressive), 20,000-24,000 (receptive) \\
\hline 12 & 8,000 - (expressive), 60,000 (receptive) \\
\hline
\end{tabular}

Table 1: Exponential growth in vocabulary during child development, potentially indicating an exponential growth in the conceptual space during development. 
If as proposed [4], [10] the location of a concept in conceptual space also correlates with the physical location in the brain, then the volume of the human brain would also be expected to be correlated with the size of the conceptual space. An exponential increase in the size of the conceptual space during a child's development should then be accompanied by an exponential increase in the size of the brain. The predicted increase in brain volume during this developmental stage is indeed what appears to be observed. Because adult humans are so encephalized, human babies have the smallest relative brain size of all of the primates, with brains less than $30 \%$ of adult size. As a result, although human newborns are precocial in other respects, our neonates are neurologically and behaviorally altricial [15] - [17]:

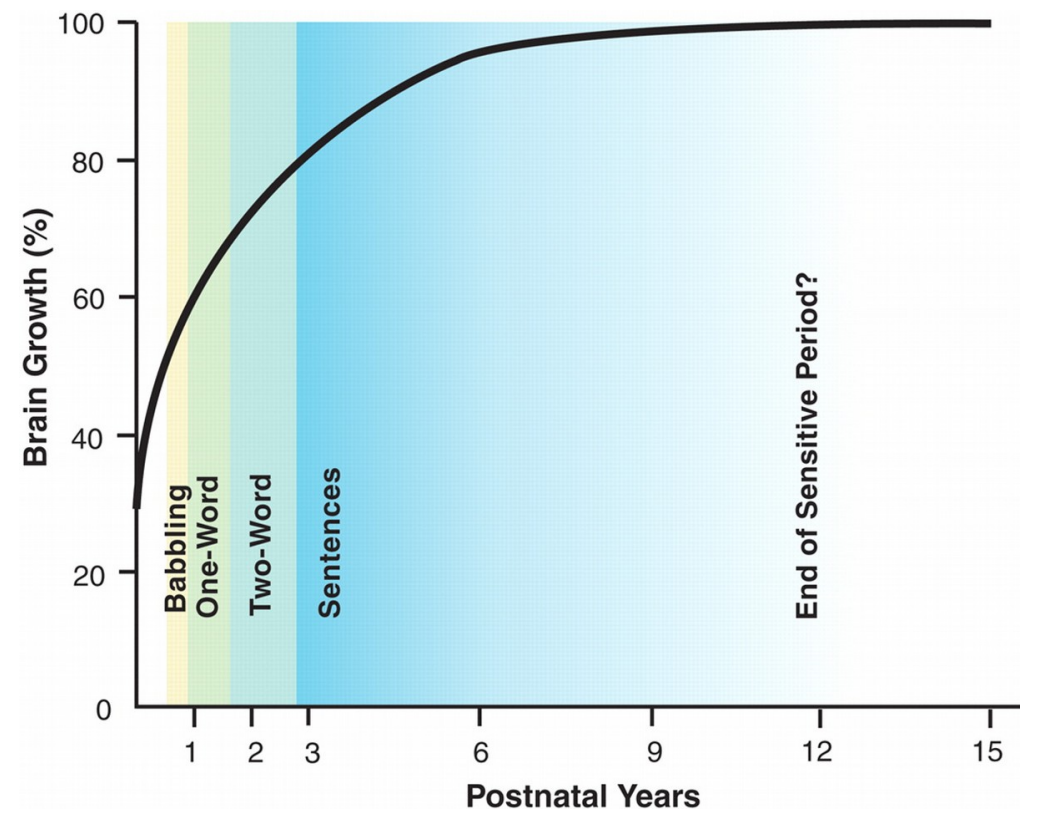

Figure 5: Postnatal human brain growth.

\section{Human Language and Scaling the Collective Conceptual Space with Functional Modeling}

When considering the minimum number of reasoning processes that must be implemented in the conceptual space of a functional model of cognition in order for an implementation of that cognitive system to have human-like general problem-solving ability, from the point of view of this functional model, it is necessary to consider whether there is a minimum level of generalization required for a system of cognition to experience a phase change in general problem-solving ability, and what characteristics of language indicate that capacity. And for a system of group cognition such as a GCI, it is necessary to consider whether there a minimum level of generalization required for that group cognition to undergo the next phase change in general problem-solving ability, and again what additional characteristics of language indicate this additional capacity.

Human languages have the capacity to convey the concept of value as an abstraction, so that this abstraction can be exchanged and accumulated at orders of magnitude greater levels than possible for any individual. Because value has the potential to impact any problem, the ability of one organism to accumulate orders of magnitude higher value than any other individual of that species is suggested to demonstrate this phase change [1]. One example of value as an abstraction is money. Human languages can communicate the concept of money, so that money can be exchanged and accumulated in this way. 
However human languages are limited in the level of complexity they can reliably communicate. Semantic modeling (functional modeling combined with a functional model of cognition with which to interpret those models) conveys more structured information that enables far greater complexity to be communicated more accurately, so that it is possible to reliably communicate the value in a second level of abstraction (an abstraction of an abstraction). In other words, semantic modeling enables processes of generalization to be defined in a way that the value of those processes is more readily exchanged and accumulated. If human languages convey concepts and relationships between them to the degree of generalization proposed to allow the exponential growth in the conceptual space that is predicted to occur as a phase change from animal to human intelligence, then this second level of language (semantic modeling) might be required to convey abstractions of concepts and the relationships between those abstractions that is proposed to allow the exponential growth in the collective conceptual space as opposed to the conceptual space of any individual, that is predicted to occur in the phase change from individual human intelligence to General Collective Intelligence. This second level of language, expressed in terms of semantic models used to define regions of the collective conceptual space, might enable individuals to exchange and accumulate such semantic models, so the cumulative conceptual space collectively known can be increased. And when this exchange can be brokered on the behalf of each individual at orders of magnitude greater speed and scale by an AGI, the potential for this cumulative conceptual space to be exponentially larger than that of the individual is clear.

\section{Conceptual Space Too Empty for Human-Like General Problem Solving Ability}

Generalizations are predicted to be too broad (regions in conceptual space include too many concepts) to be useful in problem-solving based on rational methodical reasoning within a given conceptual space if generalizations including $\mathrm{N}$ concepts don't also define a relationship between each of those $\mathrm{N}$ concepts and the generalization itself. Or in other words, in order to be useful in problem-solving a generalization containing $\mathrm{N}$ concepts must also contain $\mathrm{N}$ relationships. A cognitive system with a conceptual space that is too empty might not contain a sufficient number of concepts in each generalization for the generalization to significantly increase general problem-solving ability.

However, intuitive reasoning simply requires defining an imprecise generalization, though this imprecise generalization might not be usable in solving any specific problem. For example the concept of a "car" is a generalization that might contain many specific vehicles. But it is not until that generalization includes the description of how to drive an automatic or manual transmission car, and is is not until that generalization includes the description of the rules of the road dictating how that vehicle must be operated, that we can solve the problem of executing the functions of that generalized car.

\section{Minimum Required Number of Generalizations in the Conceptual Space for Human-Like General Problem Solving Ability}

If one generalization contains $\mathrm{M}$ concepts, and another generalization contains $\mathrm{N}$ concepts, then each potential relationship between the $\mathrm{M}$ concepts and the $\mathrm{N}$ concepts is a solution (path through conceptual space) providing a potential relationship between the generalizations. If $\mathrm{M}<=\mathrm{N}$, then the number of potential solutions between generalizations $M$ and $N$ are $M^{2}<=M^{*} N<=N^{2}$. In order to have the capacity to generalize to some degree " $\mathrm{N}$ " (to create a generalization containing $\mathrm{N}$ concepts), the cognitive system must have the capacity to define relationships between the general category and all $\mathrm{N}$ entities it contains. The total number of reasoning paths required to navigate from all the starting concepts, to the initial generalization, through all the relationships between generalizations to the final generalization, to all the ending concepts, is $\mathrm{N}+\mathrm{N}^{*} \mathrm{M}+\mathrm{M}$. 


\section{Generalization Multiplies the Number of Solutions in Conceptual Space \\ Final \\ $\mathrm{M}$ Concepts}

In order to have the capacity to reliably form these $\mathrm{N}$ relationships between the $\mathrm{N}$ concepts in the generalization and the generalization itself, the cognitive system must have the capacity to either form those relationships sequentially or in parallel. If sequentially, then for each relationship the cognitive system must have the capacity to chain together an arbitrarily long set of reasoning processes until it forms that relationship, and in order to use that relationship it must have the ability to either retrace that path once it is defined, or it must have the ability to store a direct relationship between the initial and final concept that might be used to retrieve that path. 


\section{Storing Simplest Representations of Relationships in Generalization}
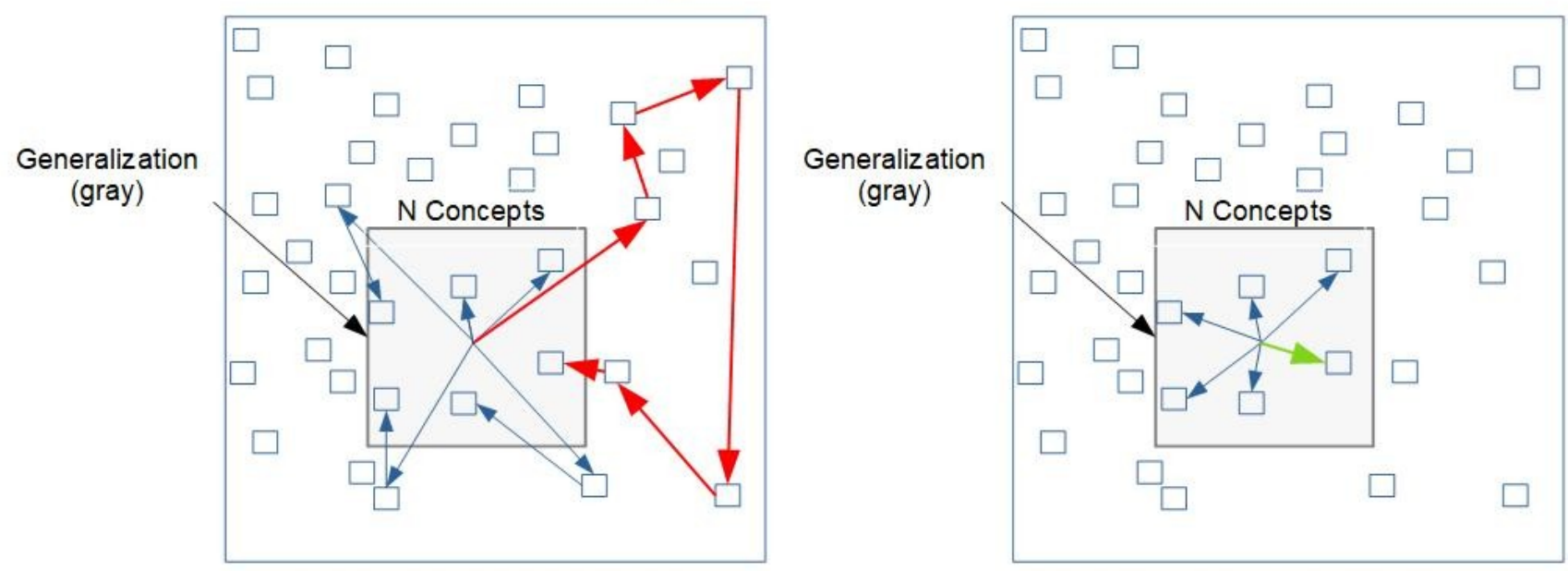

Figure 7: The reasoning defining the relationship between each concept and a generalization containing those $N$ concepts can involve many intermediate concepts (left), or it can be direct (right). A complex reasoning process (left - red) might be linked in memory to a direct relationship (rightgreen).

If in parallel (i.e. simultaneously), the cognitive system must have the capacity for that simultaneous detection of all $\mathrm{N}$ relationships. However, detecting a relationship in conceptual space or in any other functional state space, where that detection requires the simultaneous detection of $\mathrm{N}$ relationships, is a task requiring the ability to detect one possibility out of a potentially vast number of possibilities. From the functional modeling perspective, a relationship is a path from one concept (one point in conceptual space) to another. And each point (each concept) might represent a generalization that can represent multiple concepts. If VALUES $S_{i}$ is the number of values of some concept " $i$ ", then detecting relationships between $\mathrm{N}$ entities simultaneously requires the ability to detect $\mathrm{N}^{\mathrm{VALUES}}$ combinations. This can quickly become a sufficiently large number that it is likely to be unmanageable within cognitive resources.

While we cannot assume the ability to navigate $\mathrm{N}^{\text {VALUES }}$ potential reasoning processes within available cognitive resources, we assume that at least $\mathrm{N}^{2}$ reasoning processes are required for general problemsolving ability. And if human-like general problem-solving ability is an exponential increase in general problem-solving ability in any given size of conceptual space, then once the ability to chain reasoning together has been created, and once a sufficient number of points in conceptual space have been defined, then we can assume the cognitive system can increase the number $\mathrm{N}$ of concepts contained in a generalization (to the limits of memory) to the point at which the number of reasoning solutions increases to $\mathrm{N}^{3}$. Here "sufficient" means a sufficient number of generalizations so that the entire conceptual space can potentially be navigated.

Through a visual representation of the conceptual space the cognitive system can be seen to have sufficient reasoning processes to display the ability to potentially solve any general problem when the density of known relationships in conceptual space is uniform (known relationships are most evenly distributed), and the number of relationships is greater than or equal to the number of concepts $\mathrm{N}$. 


\section{Sufficient Reasoning to Solve any General Problem?}

When the cognitive system can chain reasoning in sequences and can store a sufficient number of these relationships to generalize through defining a category containing $\mathrm{M}$ concepts, then when $\mathrm{M}$ is large enough that reasoning becomes isomorphic (the beginning and end concepts remain within the same generalization), an exponential increase in the number of generalizations available, and therefore the potential for an exponential increase in the ability to generalize, occurs. This point has been theorized to be a phase change [5] at which the cognitive system might display human-like general problem solving ability if the number of concepts in the generalization and the density of concepts in the conceptual space are sufficient for general problem-solving ability at all. 


\section{Generalization to Point of Isomorphism Exponentially Multiplies Solutions in Conceptual Space}

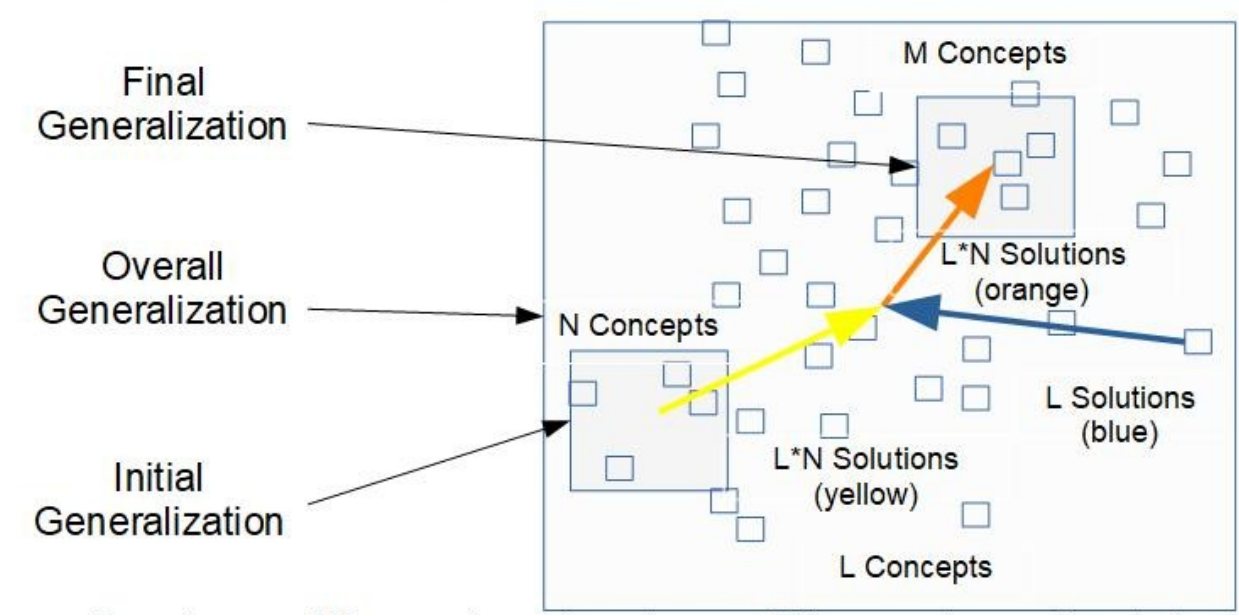

Figure 9: If a category of $N$ concepts and a category of $M$ concepts are all part of a generalization of $L$ concepts, then all L direct relationships to the overall generalization can potentially be applied to each of the $M$ and $N$ concepts, leading to a total of $M^{4}<=L^{*} L^{*} M^{*} N<=N^{4}$ relationships between $N$

If the total number of reasoning processes required in human cognition to connect $\mathrm{N}$ concepts in human-like reasoning is at minimum on the order or $\mathrm{N}^{3}$, then defining a human-like conceptual space of $20 \mathrm{~K}$ concepts requires on the order of $6^{*} 10^{12}$ reasoning processes, or some lower number of reasoning processes representing reasoning generalizations capable of navigating between generalizations of those concepts. Assume that each concept generalization on average contains $\mathrm{N}$ concepts and $\mathrm{N}$ reasoning processes linking those concepts to the concept generalization. Then spanning a human-like conceptual space of $20 \mathrm{~K}$ concepts requires at minimum (which in turn assumes none of the generalizations overlap) $20 \mathrm{~K} / \mathrm{N}$ generalizations.

These generalizations will be connected by at minimum $20 \mathrm{~K} / \mathrm{N}$ relationships. However, concepts can be linked by rational methodical reasoning or intuitive reasoning. Rational methodical reasoning processes are reducible to a minimal set of reasoning processes from which all such reasoning is composed. If the direct link between one concept and another is not specified by any single reasoning process in that minimal set, then those concepts are not directly linked by any rational methodical reasoning process. In that case, using rational methodical reasoning to define the relationship between any two concepts might require many more steps. If $\mathrm{M}$ is the maximum number of steps in a relationship that might be remembered (i.e. the maximum number of steps in a reasoning process connecting any two concepts that might be remembered), then even if only some of those processes are valid, the minimal number of reasoning processes required for human-like general problem-solving ability might be much higher. Further analysis is required to validate this speculation. Intuitive reasoning on the other hand, can potentially always be used to define direct relationships between any two concepts. Because from the functional modeling perspective intuitive reasoning is pattern matching by a black box. A pattern can always be found by such a black box. And any pattern is a solution to the problem of the lack of a relationship. However, storing such direct relationships, rather than storing minimal reasoning that can be used to deduce those relationships through rational methodical 
reasoning, might significantly increase the minimum memory resources required for the cognitive system to function.

Assuming that the minimum number of generalizations required to achieve human-like general problem-solving ability is the number at which reasoning becomes isomophic, that number might then be estimated by first dividing the conceptual space into the minimum number of regions of generalization within which isomorphism might be demonstrated. The minimum number of regions across which generalization to the point of isomorphism might be generated is two, with one region containing $\mathrm{M}$ concepts and the other containing $\mathrm{N}$ concepts. Then the number of direct relationships that a process of generalization to the isomorphism can be applied to is $\mathrm{N}^{*} \mathrm{M}$. In addition there are $\mathrm{N}$ relationships between the concepts in the generalization containing the $\mathrm{N}$ relationships, and that generalization itself. And there are $\mathrm{M}$ relationships between the concepts in the generalization containing the $\mathrm{M}$ relationships, and that generalization itself. Adding these $\mathrm{M}$ and $\mathrm{N}$ relationships to each of the generalizations results in a number of generalizations $M^{*} \mathrm{~N}+\mathrm{M}+\mathrm{N}$ that provide the ability to navigate the entire conceptual space with reasoning that includes generalization to the point of isomorphism, which according to [5] is suggested to be human-like general problem-solving ability. If $\mathrm{M}+\mathrm{N}$ is equal to $20 \mathrm{~K}$ concepts, then assuming that $\mathrm{M}=\mathrm{N}$, the ability to generalize (total number of generalizations) required for human-like general problem-solving ability is approximately $4 * 10^{8}$. Since every generalization can potentially replace $\mathrm{M}^{*} \mathrm{~N}$ specific reasoning processes that would otherwise be used to navigate between concepts, this potentially reduces the number of reasoning processes required to implement human-like AGI from being on the order of $6^{*} 10^{12}$ reasoning processes, to being on the order of $2 * 10^{4}$ reasoning processes.

Generalizations must meet criteria in addition to just containing the initial and final concepts in order for them to be usable in solving a given problem. From a functional modeling perspective, the generalization must contain the specific function defined by the problem. For example, the problem of getting a 2020 Mercedes-Benz E-Class sedan one is driving to turn right is not solved in a useful way by the generalization that it is a thing. And that a thing in some state has a process that can be executed on it to change that thing to another state. In order for the generalization to be useful, the category of generalization used to describe the 2020 Mercedes-Benz E-Class sedan must have the function of being drivable and steerable, such as the category "car".

\section{Defining a Minimal Functional Model of an AGI and Estimating the Time Required to Implement it}

If, as proposed earlier in this paper, the number of reasoning processes required to achieve human-like general problem solving ability is at minimum on the order of the cube of the number of concepts $\mathrm{N}$, and if $\mathrm{N}$ is approximately $20 \mathrm{~K}$, then having the capacity to reliably achieve human-like general problem-solving ability requires having the ability to reliably increase the number of unique reasoning processes that can be navigated until it is greater than this number $\mathrm{N}^{3}$ or $8 * 10^{12}$. However, as mentioned earlier in this paper, the designer's capacity to form relationships between these reasoning processes and every new concept introduced into the AGI, so that all concepts remain navigable, limits the size of the conceptual space to 870,000 reasoning processes $\left(8.7 * 10^{5}\right)$ or lower.

One way to achieve the capacity for the needed number of reasoning processes is to reduce the size of the problem. In order to reduce the complexity of the problem of comparing all reasoning processes (comparing all components of all AI and AGI solutions), so that converging on the functionality required for a working AGI is reliably achievable, it is necessary to minimize the number of functional models required to do so by introducing the capacity to create a number of generalizations on the order of approximately $8 * 10^{12}$, and by introducing the capacity to form relationships between these 
generalizations and every new concept introduced into the AGI at a rate sufficient for all concepts to be navigable. This paper has attempted to provide an argument that the capacity to create this number of generalizations can reliably be achieved through use of functional modeling combined with a functional model of cognition that together define semantic models, to allow semantic models to be exchanged at far greater speed and scale.

HCFM differs from other methods of functional decomposition in choosing functional components in terms of their fitness in achieving some outcome, rather than choosing functional components in terms of any intellectual theory. One intellectual theory (e.g. one model of AGI such as NARS) might not be easily comparable in terms of the concepts defined by another (e.g. another model of AGI such as ACTR). However, because all reasoning can potentially be compared directly to any other reasoning through the degree to which it functions to achieve impact on a common outcome, HCFM makes this comparison simple enough (of low enough order of complexity) to reliably be achieved. Because the comparison can be reliably achieved, and because capacity for generalization can be reliably increased to the point that such comparison can reliably be made at the rate required to accommodate new concepts generated by the reasoning process, then according to this functional model of cognition, a cognitive system meeting these minimal parameters might be expected to have the capacity for humanlike general problem-solving ability.

The functional model of cognition on which these estimates are based is proposed to be the first functional model of cognition that is complete enough to have the potential for human-like general problem-solving capacity. Having what is proposed to be a complete functional model also introduces another issue, and that is estimating the time required to achieve AGI [8], [9]. AGI, as construed by this functional model, may be implemented in software. And all known software estimation methods rely on some model of some methodology that is proposed to have the capacity to reliably result in the outcome of delivering the target functionality. However, since no other functional models of cognition have been proposed to be complete, then no estimate based on such an incomplete model can be anything other than a guess. This problem of estimation without a model of functionality that is proposed to be complete is akin to asking "how long will it take to get there?" where the destination has not been specified as even being anywhere on the planet. The answer might be anywhere from zero seconds to infinity.

\section{Using GCI to Achieve Exponential Impact on Deployment of AGI, AI, Blockchain, or Other Technology Outcome}

General problem-solving ability, as mentioned, implies the ability to impact any general problem within the capacity of the system of cognition. One general problem might be increasing the deployment of AI solutions, or increasing the deployment of blockchain technology related to the deployment of AI solutions. If each potential instance of an implementation of an AI solution, or each potential instance of an implementation of a blockchain technology solution (i.e. each deployment) is a concept, then reliably achieving an exponential increase in deployment of such concepts requires either an exponential increase in ability to create concepts per entity. Or it requires an exponential increase in the number of entities creating concepts. If, as predicted, GCI represents a phase change in intelligence that results in an exponential increase in problem-solving ability as compared to the ability of any individual, and if general problem-solving ability is the capacity to navigate the conceptual space to new concepts, and therefore the capacity to create new concepts, then an exponential increase in general problem-solving ability equates to an exponential ability to create concepts. If so, then GCI represents one potential way to gain the capacity to reliably achieve an exponential increase in the deployment of technologies such as AI, or blockchain. This capacity is being explored in conceptual case studies to be validated through the survey referred to in this paper [Reference to survey described 
in section "Consensus Validation of the Impact and Importance of Defining and Implementing a General Collective Intelligence to Artificial General Intelligence and Artificial Intelligence”].

\section{Scaling Cooperation to Gain Needed Capacity for Complexity}

In order to increase the number of AI and AGI researchers and developers participating in defining functional models of their solutions so that as the number of the functional models of those solutions and their components increases, the collective effort might converge on the functionality required for AGI, it is necessary to provide compelling incentive. One set of incentives is that if for every other implementation to which a given researchers work applies, that researchers work gains some minimal additional value $\mathrm{V}$, then defining this kind of a common framework that creates the potential to exponentially increase the number of those implementations, will also increase the visibility, impact, revenue, and other outcomes associated with each implementation.

In order to manifest this potential, one strategy might be to define a software application that uses a common functional modeling approach to represent an AI solution, and that uses a common approach to generalizing that functional model so it applies to many more problems. Then one might identify $\mathrm{N}$ problems that any given AI solution might be applied to, and algorithmically search for all combinations of functional models of AI solutions that are projected to solve a given problem, as well as search for all the AI solutions implementing those functional models, and determine the projected or actually relative fitness of each solution in achieving an outcome related to that problem. By awarding each AI or AGI researcher some portion of the value $\mathrm{V}$ that their component added to this new solution opportunity, and by greatly increasing the number of solution opportunities in this way, outcomes for each researcher might be increased to the point that they might reliably be incentivized to participate. In this way GCI might be used to organize all AI and AGI researchers to cooperation in searching all potential designs for an AGI, to find the design that functions best.

\section{Can a Functional Modeling Approach Reliably Implement Artificial General Intelligence?}

Any single proposed design for an AGI can potentially be incapable of demonstrating human-like general problem-solving ability. However, the functional modeling approach combined with GCI is not a single design. It's essentially a methodology for exploring all possible designs in a way that is intended to converge on whatever design achieves maximum fitness in executing the functionality required for an AGI with human-like intelligence. And GCI is intended to do so at exponentially greater speed and scale that possible otherwise. Any functional component of the approach including the functional model of cognition itself, can potentially be replaced by one that is more fit.

In determining whether the combination of functional modeling and GCI can reliably implement an AGI, one potential concern is ensuring the design process doesn't get stuck in local maxima of fitness. For example, a given functional component in the design might perform optimally with a given subcomponent. But the optimal design might be to eliminate that component entirely. This problem is potentially avoidable by assessing fitness at every component or sub-component level, thereby enabling every component at every level to be replaced by one that is more fit. Since the functional model is the top-level component, the fact that the functional model itself can be replaced would appear to be a safeguard against this problem.

\section{Consensus Validation of the Impact and Importance of Defining and Implementing a General Collective Intelligence to Artificial General Intelligence and Artificial Intelligence}

As mentioned, a GCI that enables groups to reason collectively has the potential to drive an exponential increase in general problem-solving ability over any individual in the group [a]. In order to achieve this potential, a GCI enables the group to abstract all concepts so that the value of any concept in solving 
any other problem can be more easily determined. Since impact on a problem is value, the resulting ability of the group to exchange and accumulate impact on any general problem creates the potential for this significant increase in problem-solving ability. This significant increase rises to the point of being an exponential one when the level of abstraction results in the initial and final concepts falling within the same generalization so that reasoning becomes isometric within the same space. At this point the solutions available to collective cognitive system increase exponentially. The visual representation of this suggested phase change in the conceptual space has been explored [5].

In order to achieve this exponential increase in human general problem-solving ability, all information must be modeled in a common way. This common way is as constructs in the collective conceptual space. Because GCI has been been suggested to be, by value of potential impact, indisputably the most important innovation in the world today by a large margin [5], then if defining and implementing a conceptual space is critical to GCI, that makes doing so more important than any other endeavor. If modeling in terms of constructs within the conceptual space is a modeling approach, this makes it the most important modeling approach in the world today. However, the theory behind GCI suggests that this cannot be proven. GCI defines patterns of organization that allow groups to self-assemble solutions, rather than any individual in the GCI defining each solution themselves as a specific implementation of those patterns. Because groups self-assemble solutions, the specific solutions implementing those patterns can't be known ahead of time.

Because the specific mechanism by which GCI might increase impact on any general problem, and why that increase might be exponential, cannot be known beforehand by any individual or by any group without GCI, GCI based solutions can only be described in a way that is too general to be provable. As a result, the potential of GCI to increase impact cannot be proved to anyone without GCI. Just as with AGI as explored at the beginning of this paper, in the case of sufficiently complex concepts, which higher order systems like an implementation of AGI or GCI are by definition, no one individual can reliably communicate that concept in a way that other people can reliably understand. To understand GCI, one must first believe this general concept might be true, so one can then convince oneself of the truth of it over time by verifying this generalization is true within every testable instance, and therefore is likely true in all instances. Even though the mechanism by which GCI might exponentially increase impact on a specific problem cannot be known beforehand, by seeing that it always does, one can slowly gain confidence that GCI is observed to have the ability to do so in all cases. The design of a collective intelligence is also too complex for any individual human to understand and communicate at a specific enough level to reliably be able to convince the number and diversity of expert roles required in order to build one. Solving "higher order complexity" problems requires GCI. Therefore solving the higher order complexity problem of launching a project to build a General Collective Intelligence in order to test the impact of one, requires a General Collective Intelligence itself.

The solution to this riddle might involve an evolutionary approach, building whatever small segment of GCI can be built by an individual or group without GCI, to test the impact of GCI on that problem, and then using that group and its mind share, as well as that GCI functionality, to generate resources to engage a larger group of more specialized individuals to build more GCI functionality and to test it's impact on a more general problem faced by that group, doing so in a cycle until GCI is built, and GCI's potential to increase a group's general problem-solving ability has been proved so that GCI can be used to orchestrate massive cooperation, that in turn might direct massive resources towards actually achieving that general problem-solving ability. 
This evolutionary approach is currently being used in an iterative series of surveys to demonstrate a consensus of expert opinion in the AI and AGI communities that this GCI based approach has the potential to significantly increase impact on a specific program, where that problem is broadened with each iteration of the survey, until consensus that this GCI based approach has the potential to significantly increase impact on the general problem of AGI among all relevant expert communities is demonstrated.

Two sets of surveys are envisioned to be performed. As described in the tables below, the specific problems that the initial survey in the first set will target is that GCI has the potential to significantly increase impact on solving the problem of learning from each approach to AGI in a way that increases the probability of actually implementing a working AGI. This consensus is intended to be demonstrated through a series of GCI based surveys. Each survey in this series is designed to build mind share and to use GCI to adapt to increase that mind share among a larger and more diverse range of more specialized experts in the next iteration, while also gaining financial resources to incentivize those experts to invest more time contributing to that next iteration, until enagement of any parties required to overcome any visible or invisible hurdles to achieving the targeted outcome (in this case demonstrating the importance of modeling in terms of constructs in the conceptual space), is reliably achievable. This survey begins with the general community interested in AGI, and then evolves to engage the research community specializing in AGI through academic conferences targeting those communities. To demonstrate the impact on the general problem of implementing a working AGI, rather than choosing to prove the impact on any specific approach to AGI, the conferences targeted were simply chosen by selecting the most popular results returned from a Google search for the term "AGI conference".

As described in the same table, the specific problems that the initial survey in the second set will target is that GCI has the potential to significantly increase impact on solving the problem of learning from each approach to AGI in a way that increases the probability of actually implementing a working AGI. This survey is intended to evolve into demonstrating consensus that GCI can reliably achieve a significant increase in impact on the general problem of achieving an exponential increase in deployment of AI solutions. To demonstrate the impact, the AI experts to survey were simply chosen by targeting the most popular results returned from a Google search for the term "AI conference".

\section{Conference}

AGI 2021

AAAI 2021

\section{Claim to be Proved}

GCI has the potential to significantly increase impact on solving the problem of implementing a working AGI.

GCI has the potential to significantly reliably achieve an exponential increase in deployment of AI solutions.

Table 3: AI and AGI conferences and claims to be explored through survey.

This iterative survey approach is also being applied to other areas, such as the modeling required for both GCI and AGI. The specific problem that the initial survey will target in the case of modeling is that modeling in terms of constructs in the collective conceptual space has the potential to significantly increase impact on the targeted outcomes of modeling as compared to any other modeling approach. This consensus is intended to be demonstrated through a series of GCI based surveys. Each survey in this series is designed to build mind share and to use GCI to adapt to increase that mind share among a larger and more diverse range of more specialized experts in the next iteration, while also gaining financial resources to incentivize those experts to invest more time contributing to that next iteration, 
until enagement of any parties required to overcome any visible or invisible hurdles to achieving the targeted outcome (in this case demonstrating the importance of modeling in terms of constructs in the conceptual space), is reliably achievable. This survey begins with the general community engaged in any modeling effort of any kind, and then evolves to engage specialized modeling communities through academic conferences targeting those communities. To demonstrate the impact on the general problem of modeling, rather than choosing to prove the impact on any specific category of modeling, the modeling experts to survey were simply chosen by selecting the most popular results returned from a Google search for the term "modeling conference".

\section{Validating the Completeness and Correctness of the Functional Model of Cognition}

The theory of HCFM suggests that solutions to higher-order problems such as cognition cannot be proven within the capacity of individual human cognition. Instead one must decouple the problem into many lower order ones, and prove the solution to each one. Accordingly, the next step in engaging with any AGI researcher is designing an experiment to prove that the "change engine" model of cognition defined within the FMF using the HCFM approach could be used to represent the researcher's model of AGI. For example, in the case of the NARS model for AGI. To do this, an AGI model must be decoupled into functional components using the functional model of cognition. This is as simple as using the NARS AGI model to connect any two points in conceptual space, as an implementation of the corresponding reasoning process in the functional model. And it's as simple as using the NARS AGI model to implement the awareness process of the functional model. If the NARS model or other of AGI can be confirmed to still work despite having been decoupled into these functional components, this might provide confirmation that the decomposition of artificial cognition into these functional components allows the model of cognition to incorporate the functionality in at least this one (NARS) model of AGI.

\section{Conclusions}

From the perspective of HCFM, the number of reasoning processes required for an AGI to demonstrate human-like general problem-solving ability has been estimated to be on the order of . And the number of processes of generalization required has been estimated as being on the order of $4 * 10^{8}$. Without an AGI these processes must be manually modeled in the conceptual space by human-beings, in order for them to be accessible to the AGI. Without GCI to define a collective conceptual space in which functional models of concepts can be defined, and without GCI to introduce a collective system of cognition in which those functional models have meaning, the level of generalization required to make this manual modeling achievable at all, much less reliably achievable, cannot be attained. Furthermore, no other existing model of AGI defines a complete functional model of cognition. So no other existing model of AGI can identify a path to reliably achieving AGI. Therefore, according to this reasoning GCI, is required in order to reliably achieve AGI.

\section{References}

[1] Williams, A. E. (2020, April 16). A Human-Centric Functional Modeling Framework for Defining and Comparing Models of Consciousness and Cognition. https://doi.org/10.31234/osf.io/94gw3 [2] Williams, A. E. (2020, May 15). Use of Human-Centric Functional Modeling to Maximize Convergence in Integrative Research. https://doi.org/10.31730/osf.io/jv6h8

[3] Williams, A. E. (2020, April 27). Defining Functional Models of Artificial Intelligence Solutions to Create a Library that an Artificial General Intelligence can use to Increase General Problem Solving Ability. https://doi.org/10.31730/osf.io/hpzb7

[4] Andy E. Williams et al, Defining and Implementing a Conceptual Space as the Functional State Space of the Cognitive System in a Human-Centric Modeling Framework, working paper (2020) 
[5] Williams, A. E. (2020, July 11). Human Intelligence and General Collective Intelligence as Phase Changes in Animal Intelligence. https://doi.org/10.31234/osf.io/dr8qn

[6] Brysbaert M, Stevens M, Mandera P and Keuleers E (2016) How Many Words Do We Know?

Practical Estimates of Vocabulary Size Dependent on Word Definition, the Degree of Language Input and the Participant's Age. Front. Psychol. 7:1116. doi: 10.3389/fpsyg.2016.01116

[7] Dutka, Jacques (1991), "The early history of the factorial function", Archive for History of Exact Sciences, 43 (3): 225-249, doi:10.1007/BF00389433

[8] Seth D. Baum, Ben Goertzel, Ted G. Goertzel, How long until human-level AI? Results from an expert assessment, Technological Forecasting and Social Change, Volume 78, Issue 1, 2011, Pages 185 195, ISSN 0040-1625, https://doi.org/10.1016/j.techfore.2010.09.006.

[9] Vincent C. Müller and Nick Bostrom. 2014. Future progress in artificial intelligence: a poll among experts. AI Matters 1, 1 (August 2014), 9-11. DOI:https://doi.org/10.1145/2639475.2639478 [10] Günther, F., Rinaldi, L., \& Marelli, M. (2019). Vector-Space Models of Semantic Representation From a Cognitive Perspective: A Discussion of Common Misconceptions. Perspectives on Psychological Science, 14(6), 1006-1033. https://doi.org/10.1177/1745691619861372 [11] Arel I. (2012) Deep Reinforcement Learning as Foundation for Artificial General Intelligence. In: Wang P., Goertzel B. (eds) Theoretical Foundations of Artificial General Intelligence. Atlantis Thinking Machines, vol 4. Atlantis Press, Paris

[12] Pennachin C., Goertzel B. (2007) Contemporary Approaches to Artificial General Intelligence. In: Goertzel B., Pennachin C. (eds) Artificial General Intelligence. Cognitive Technologies. Springer, Berlin, Heidelberg

[13] Eliezer Yudkowsky, Levels of organization in general intelligence, Artificial General Intelligence, 2005, Springer-Verlag

[14] Thórisson K.R., Bieger J., Thorarensen T., Sigurðardóttir J.S., Steunebrink B.R. (2016) Why Artificial Intelligence Needs a Task Theory. In: Steunebrink B., Wang P., Goertzel B. (eds) Artificial General Intelligence. AGI 2016. Lecture Notes in Computer Science, vol 9782. Springer, Cham [15] Kuniyoshi L. Sakai, Language Acquisition and Brain Development, Science 04 Nov 2005: Vol. 310, Issue 5749, pp. 815-819, DOI: 10.1126/science.1113530

[16] Dunsworth, Holly M. "Thank your intelligent mother for your big brain." Proceedings of the National Academy of Sciences of the United States of America vol. 113,25 (2016): 6816-8. doi:10.1073/pnas.1606596113

[17] Dunsworth, Holly M et al. "Metabolic hypothesis for human altriciality." Proceedings of the National Academy of Sciences of the United States of America vol. 109,38 (2012): 15212-6. doi:10.1073/pnas.1205282109

[18] Nielsen F.Å., Hansen L.K. (2020) Creating Semantic Representations. In: Sikström S., Garcia D. (eds) Statistical Semantics. Springer, Cham. https://doi.org/10.1007/978-3-030-37250-7_ 